Accepted refereed manuscript of:

Hoyle A, Bowers R, White A \& Boots M (2008) The influence of trade-off shape on evolutionary behaviour in classical ecological scenarios, Journal of Theoretical Biology, 250 (3), pp. 498-511.

DOI: $10.1016 /$ j.jtbi.2007.10.009

(C) 2007, Elsevier. Licensed under the Creative Commons AttributionNonCommercial-NoDerivatives 4.0 International http://creativecommons.org/licenses/by-nc-nd/4.0/ 


\section{The influence of trade-off shape on evolutionary behaviour in}

\section{2 classical ecological scenarios}

3

4 Andrew Hoyle ${ }^{1,5,9}$, Roger G. Bowers ${ }^{2,6}$, Andrew White ${ }^{3,7}$ and Michael Boots ${ }^{4,8}$

5

$6{ }^{1}$ Department of Computing Science and Mathematics, University of Stirling, Stirling,

7 Scotland, FK9 4LA, UK.

$8{ }^{2}$ Department of Mathematical Sciences, Division of Applied Mathematics,

9 Mathematical Sciences Building, The University of Liverpool, L69 7ZL, UK.

$10{ }^{3}$ Department of Mathematics and the Maxwell Institute for Mathematical Sciences,

11 Heriot Watt University, Edinburgh, EH14 4AS, UK.

12

13 Bank, Sheffield, S10 2TN, UK.

14

$15 \quad{ }^{5}$ Email: A.Hoyle@maths.stir.ac.uk

16 Email: sx04@liv.ac.uk

$17 \quad{ }^{7}$ Email: A.R.White@hw.ac.uk

$18 \quad{ }^{8}$ Email:M.Boots@sheffield.ac.uk

$20 \quad{ }^{9}$ Corresponding author

21 Tel: $+44(0) 1786467467$,

22 Fax: $+44(0) 1786464551$

23

24 
1 Abstract - Trade-off shapes are crucial to evolutionary outcomes. However, due to

2 different ecological feedbacks their implications may depend not only on the trade-off

3 being considered but also the ecological scenario. Here, we apply a novel geometric

4 technique, trade-off and invasion plots (TIPs), to examine in detail how the shape of

5 trade-off relationships affect evolutionary outcomes under a range of classic

6 ecological scenarios including Lotka-Volterra type and host-parasite interactions. We

7 choose models of increasing complexity in order to gain an insight into the features of

8 ecological systems that determine the evolutionary outcomes. In particular we focus

9 on when evolutionary attractors, repellors and branching points occur and how this

10 depends on whether the costs are accelerating (benefits become 'increasingly' costly),

11 decelerating (benefits become 'decreasingly' costly) or constant. In all cases strongly

12 accelerating costs lead to attractors while strongly decelerating ones lead to repellors,

13 but with weaker relationships, this no longer holds. For some systems weakly

14 accelerating costs may lead to repellors and decelerating costs may lead to attractors.

15 In many scenarios it is weakly decelerating costs that lead to branching points, but

16 weakly accelerating and linear costs may also lead to disruptive selection in particular

17 ecological scenarios. Using our models we suggest a classification of ecological

18 interactions, based on three distinct criteria, that can produce one of four fundamental

19 TIPs which allow for different evolutionary behaviour. This provides a baseline

20 theory which may inform the prediction of evolutionary outcomes in similar yet

21 unexplored ecological scenarios. In addition we discuss the implications of our results

22 to a number of specific life-history trade-offs in the classic ecological scenarios

23 represented by our models.

25 Keywords.- Trade-offs, shapes, evolution, frequency dependence, TIPs. 


\section{Introduction}

3 Life history theory has long recognised the importance of trade-offs in determining

4 evolutionary behaviour (see Stearns, 1992; Roff, 2002 for reviews). It is also

5 increasingly recognised that the shape of trade-offs, in addition to the level of costs, is

6 crucial in determining the evolutionary dynamics (see Levins 1962, 1968; de

7 Mazancourt and Dieckmann, 2004; Rueffler et al., 2004; Bowers et al., 2005). By

8 definition, in all trade-off relationships, benefits in one life-history trait come at a cost

9 in terms of another component of fitness. In general then as benefits through one trait

10 increase, the costs due to the change in the other trait may increase at the same rate,

11 leading to an exactly linear trade-off; alternatively the costs may accelerate (increase

12 quicker than the benefits) or decelerate (increase slower than the benefits), so that

13 benefits become increasingly or decreasingly costly. When the benefits of a trait are

14 met with accelerating costs in the correlated trait we define an 'acceleratingly costly

15 trade-off'. Conversely we define 'deceleratingly costly trade-offs' when the costs

16 decelerate. Here our aim is to understand how these different shapes of trade-offs

17 influence evolutionary outcomes in a range of scenarios described by a number of

18 classic ecological models.

The importance of the shape of trade-off relationships was first made clear in

21 the work of Levins $(1962,1968)$. He developed a graphical technique that plots the

22 fitness landscape from the fitness contours for two traits onto which the trade-off

23 relationship between them is superimposed. Applying these techniques to the

24 evolution of reproductive effort it was shown that the optimal strategy for a trade-off

25 with decelerating costs is at the maximum reproductive effort whereas for a trade-off 
1 with accelerating costs it is at an intermediate state (see Stearns, 1992). However,

2 optimisation approaches, such as Levin's are not appropriate, when there is

3 frequency-dependent (density-dependent) selection (Maynard Smith, 1982; de

4 Mazancourt and Dieckmann, 2004; Rueffler et al., 2004; Bowers et al., 2005).

5 Different ecological interactions result in particular feedbacks that may clearly lead to

6 different selection pressures on traits (Abrams, 2001) that in turn depend on the nature

7 of the trade-off connections between traits. Here we will examine how trade-off

8 shapes influence evolutionary behaviour in a number of fundamental ecological

9 models using a geometric approach that incorporates frequency-dependent selection.

10

Under frequency-dependent selection, the evolution of traits is dependent on the ecological feedbacks in the system since for a mutation to be successful it must be able to invade a population whose ecological characteristics are being determined by the resident strain (Metz et al., 1996; Geritz et al., 1998). Successful mutant invasion necessarily changes the resident and therefore also reshapes the characteristics of the population. This approach has been applied to a number of specific ecological scenarios in which trade-off relationships have been explicitly considered (Boots and Haraguchi, 1999; Kisdi, 2001; Day et al., 2002; Bowers et al., 2003; Egas et al., 2004; de Mazancourt and Dieckmann, 2004; White and Bowers, 2005; Rueffler et al., 2006). In particular, the importance of the trade-off shape in characterising evolutionary behaviour has recently been examined in detail with the development of general geometric methods for analysing the evolutionary dynamics (de Mazancourt and Dieckmann, 2004; Rueffler et al., 2004; Bowers et al., 2005). Rueffler et al. (2004) extended the Levins fitness landscape approach to allow for frequency-dependent selection for specific trade-off functions. This was further extended to enable 
1 visualisation of the effect of general trade-off functions on evolutionary outcomes (de

2 Mazancourt and Dieckmann, 2004). The method of trade-off invasion plots (TIPs)

3 developed by Bowers et al. (2005) and first used in Boots and Bowers (2004) is

4 similar to that of de Mazancourt and Dieckmann (2004) in that it is a geometric

5 technique that allows the visualisation of the role of the trade-off shape. From TIPs

6 given a specific ecological model, it is easy to determine which trade-off shapes (or

7 cost structures) produce for example, evolutionary branching. Although globally the

8 curvature of the trade-off can change sign, for example in sigmoidal trade-offs, TIPs

9 focus on the shape of the trade-off locally about the evolutionary singularity; in this

10 local region the curvature of the trade-off stays relatively constant and hence falls into

11 one of three shapes: decelerating, accelerating or straight (linear).

In this study we use TIPs to explore which ecological characteristics lead to

14 different evolutionary behaviours with trade-offs of different shapes. We use classic

15 models of increasing ecological complexity in order to reveal the ecological features

16 that underlie four fundamental types of TIPs. By classic we mean Lotka-Volterra type

17 continuous time models, i.e. where the dynamics are linear in terms of the 'evolving'

18 parameters (those involved in the trade-off) and of order one or two (linear, bilinear or

19 quadratic) in terms of densities. We choose this framework as it underpins many

20 model structures in theoretical ecology. In particular, we examine classical single

21 species models, Lotka-Volterra models that include species interactions with one class

22 of individuals for both species and host-parasite systems with multi-class interactions.

23 These TIPs in turn define all the evolutionary implications of different trade-off

24 shapes. In this way we outline how the incorporation of additional ecological

25 mechanisms can alter the topology of the invasion boundaries on a TIP and therefore 
1 also the possible evolutionary outcomes. For our range of models, we classify, by means of three criteria, the necessary general ecological characteristics and trade-off set-up required to produce different types of evolutionary behaviour. By focussing on

4 the classical models that underpin much ecological theory, our aim is to provide a

5 baseline that may inform the understanding of more complex ecological scenarios that 6 can be modelled by these type of systems.

\section{The approach: Trade-off and invasion plots (TIPs)}

12 A detailed description of the use of trade-off and invasion plots (TIPs) to determine 13 evolutionary behaviour has been given elsewhere (Bowers et al., 2005) and their 14 derivation is outlined in the appendix. An example of a TIP showing its key features 15 can be seen in Fig. 1 (where Table 5 (see Appendix) has been used to determine 16 whether a region is ES or CS). The mutant-resident invasion boundaries $\left(f_{1}, f_{2}\right)$ are 17 plotted in trait space and the trade-off line $(f)$ is superimposed such that the position

18 of the trade-off line in relation to the invasion curves determines the evolutionary

19 behaviour. The main advantage of the approach is the relatively easy way in which the

20 nature of the evolutionary outcome can be determined. Thus in Fig. 1, we can

21 immediately see that the evolutionary singularity (the point at the top right hand

22 corner of the TIP in this case, where the fitness gradient is zero) is a branching point

23 (the trait will be selected to move towards the point and then branch because the

24 trade-off curve $f$ enters the TIP in the region where $x^{*}$ is convergent stable (CS) but 25 not resistant to invasion and therefore not evolutionarily stable (ES)). The 
1 evolutionary behaviour of other trade-off curves can also be determined from the TIP.

2 For instance, if the trade-off curve entered the TIP below the mutant $\left(f_{1}\right)$ invasion

3 boundary in Fig. 1 then the evolutionary singularity would be an attractor (that is CS

4 and ES). As such the singular point is a 'continuously stable strategy' (CSS) and

5 always the end point of evolution. In contrast, if the trade-off curve entered above the

6 dashed line (mean curvature) of Fig. 1 the singularity would be a repellor (since it is

7 not CS and therefore strains further away from it invade those closer). Thus a visual

8 inspection of the TIP can indicate the type of evolutionary behaviour expected for

9 different trade-off shapes (i.e. by the direction in and strength at which $f$ curves)

10 without the need to specify the trade-off explicitly.

\section{Results}

14 We begin with a basic maturation model consisting of two stages, a non-reproducing

15 juvenile stage and a reproducing mature stage. This, for two strains $x$ and $y$, is

16 defined by the continuous time age-structured dynamics

17

18

$$
\begin{aligned}
\frac{d X_{1}}{d t} & =a_{x} X_{2}-q_{x} X_{2}\left(X_{1}+X_{2}+Y_{1}+Y_{2}\right)-b_{x} X_{1}-m_{x} X_{1}, \\
\frac{d Y_{1}}{d t} & =a_{y} Y_{2}-q_{y} Y_{2}\left(X_{1}+X_{2}+Y_{1}+Y_{2}\right)-b_{y} Y_{1}-m_{y} Y_{1}, \\
\frac{d X_{2}}{d t} & =m_{x} X_{1}-e_{x} X_{2}, \\
\frac{d Y_{2}}{d t} & =m_{y} Y_{1}-e_{y} Y_{2},
\end{aligned}
$$

20 where $X_{1}$ and $X_{2}$ denote the number of juveniles and matures, respectively, for strain

$21 x$, and similarly $Y_{1}$ and $Y_{2}$ for strain $y$. Also, $a$ represents the per capita 
1 reproduction rate of matures (offspring, of course, enter into the juvenile stage), $q$ the

2 rate of intraspecific competition where density-dependence is taken to act on births of

3 juveniles (i.e. of the form $(a-q H) X_{2}$ with $\left.H=X_{1}+X_{2}+Y_{1}+Y_{2}\right), b$ and $e$ the

4 death rates of juveniles and matures respectively, and $m$ the maturation rate which we

5 take to be directly (linearly) related to the number of juveniles.

6

7 Calculating the fitness function $s_{x}(y)$, which defines the long-term exponential

8 growth rate (Metz et al., 1992) of a mutant strain $y$, of low density, attempting to

9 invade an established resident population of strain $x$, we get

$11 s_{x}(y)=\frac{1}{b_{y}+m_{y}}\left(-b_{y}+\frac{m_{y}}{e_{y}}\left(a_{y}-q_{y}\left(X_{1}+X_{2}\right)-e_{y}\right)\right)$,

13 (see equations (A.2)-(A.6)). From the form for this, with the population equilibrium

14 densities (from equation (A.1) which we take to be stable) included explicitly

15 (equation (A.7)), and the equivalent form of $s_{y}(x)$ (found simply by switching the $x$

16 and $y$ parameters, as $y$ is now the resident and $x$ the invading mutant), we find that

17 the two fitness functions are related by $s_{x}(y)=-A s_{y}(x)$, where $A>0$. This implies

18 that whenever $s_{x}(y)$ is positive, $s_{y}(x)$ is negative, and vice-versa. Therefore one

19 strain will always 'win', with no possible co-existence between two strains as the

20 fitness functions can never both be positive simultaneously. In terms of TIPs, this has

21 the consequence that the fitness functions are always zero simultaneously, which in

22 turn, results in the invasion boundaries being identical (i.e. superimposed). From this, 
1 we can see that there will be no 'middle' region between the invasion boundaries and hence no possibility of evolutionary branching (see, for example, Fig. 2).

4 As $f_{1}$ and $f_{2}$ are identical, we will concentrate on the invasion boundary $f_{1}$,

5 stemming from $s_{x}(y)$ being zero. Firstly assuming the trade-off involves the birth rate

6 and is of the form $a=f(\cdot), f_{1}$ is, from equation (2),

$8 \quad a_{y}=f_{1}(\cdot)=e_{y}+\frac{b_{y} e_{y}}{m_{y}}+q_{y}\left(X_{1}+X_{2}\right)$

10 Taking each of the remaining four parameters in turn to be the second parameter

11 involved in the trade-off, the curvature of the invasion boundaries at the tip of a TIP

12 (and hence their shapes) can be calculated. The first observation we make is that the

13 parameter concerning maturation, $m$, is the only one that appears non-linearly. The

14 remaining parameters all appear linearly and hence for trade-offs between birth rate $a$

15 and either one of the death rates, $b$ or $e$, or the level of intraspecific competition, $q$,

16 the curvature of $f_{1}$ will be zero and hence the invasion boundaries will be straight. In

17 terms of evolutionary outcomes, this means that the singularity is an attractor for

18 accelerating trade-offs and a repellor for decelerating trade-offs. The singular TIPs for

19 these trade-offs will take the form of Fig. 2A. For these trade-offs $f^{\prime}>0$ (where

$20 a=f()$.$) as a decrease in either the death rate of the juveniles or matures, b$ or $e$, or

21 the level of the intrinsic growth rate, $q$, comes at a cost of a lower birth rate, $a$. The

22 results for the trade-off between adult birth and survival parallel the findings in

23 classical life history theory on the evolution of fecundity (Schaffer, 1974; Stearns, 
1 1992). A trade-off with accelerating costs lead to the evolution of intermediate birth

2 and death rates (because the singular point on the TIP is a CSS) whereas one with

3 decelerating costs leads to the evolution of extreme parameter values, such as

4 maximum birth and death rate (because the singular point on the TIP is a repellor).

6 The remaining choice of trade-off (taken in association with the invasion boundary in

7 equation (3)), between the adult birth rate, $a$, and the maturation rate, $m$, results in

8 invasion boundaries with positive curvature. In terms of TIPs, $f_{1}$ and $f_{2}$ curve (but

9 remain superimposed) resulting in a singular TIP of the form as in Fig. 2B. Here

$10 f^{\prime}<0$ (where $a=f(m \cdot)$ ) as an increase in the maturation rate, $m$, comes at a cost of

11 a lower birth rate, $a$. Here, evolutionary attractors occur not only for accelerating

12 trade-offs but also weakly decelerating trade-offs (and linear trade-offs), whereas

13 evolutionary repellors only occur for strongly decelerating trade-offs. Clearly the

14 relative strength of the costs depends on the relative curvatures of the invasion

15 boundaries. However in general, we use the short hand "weak" where the trade-offs

16 are relatively close to linear.

18 The results for these four choices of trade-offs considered in Fig. 2 are shown in the

19 top row of Table 1 . The letters denote whether the invasion boundaries curve in the

20 same manner as an accelerating trade-off, 'A', a decelerating trade-off, 'D', or are

21 linear/straight, ' 0 '. Since the invasion boundaries are superimposed (the $=$ denotes

22 this), these letters also record the shape of trade-off for which a singularity changes

23 from being an attractor to a repellor. For example, a 'DD' implies that a singularity

24 changes evolutionary outcome depending on the magnitude of a decelerating trade- 
1 off, i.e. a repellor for strongly decelerating trade-offs but an attractor for weakly

2 decelerating trade-offs (as in the case of the trade-off between $a$ and $m$ in Fig. 2).

4 Table 1 also shows the shape of the invasion boundaries for all the remaining possible

5 choices of trade-offs. From this we note that only a small number of trade-off choices

6 in our simple maturation model give curved invasion boundaries on TIPs. These

$7 \quad$ include the birth rate against the maturation rate $(a$ and $m$ ), the intraspecific

8 competition rate against the maturation rate $(q$ and $m$ ) and the maturation rate

9 against the death rate of mature individuals ( $m$ and $e$ ). In these cases evolutionary

10 attractors occur for accelerating trade-offs and weakly decelerating trade-offs, and

11 evolutionary repellors for strongly decelerating trade-offs. In contrast, with a trade-off

12 between the juvenile death rate and the mature death rate ( $b$ and $e$ ), evolutionary

13 attractors occur for strongly accelerating trade-offs only whereas evolutionary

14 repellors occur for both decelerating trade-offs and weakly accelerating trade-offs. All

15 the remaining possible trade-offs have linear/straight invasion boundaries, and hence

16 evolutionary attractors always occur for accelerating trade-offs and evolutionary

17 repellors for decelerating trade-offs.

18

\section{Single class, multi-species interactions}

20 In our remaining models we examine evolution in one species involved in an

21 interaction with another species. To begin with we assume that the dynamics of each

22 species can be described by a single class, the classical examples of which are

23 competition, mutualism and predator-prey. These interactions are all very similar in

24 terms of their dynamics, therefore we aim to set up and use differential equations

25 covering them all and, for predator-prey, examine in turn both prey and predator 
1 evolution. Taking two strains of our evolving species, denoted $x$ and $y$, and a single

2 strain of our non-evolving species, denoted $z$, gives the Lotka-Volterra form

3

$$
\begin{aligned}
\frac{d X}{d t} & =\delta_{1} r_{x} X-q_{x} X(X+Y)+\delta_{3} c_{x z} X Z, \\
4 \quad \frac{d Y}{d t} & =\delta_{1} r_{y} Y-q_{y} Y(X+Y)+\delta_{3} c_{y z} Y Z, \quad \text { where } \delta_{1}, \delta_{2}, \delta_{3}= \pm 1, \\
\frac{d Z}{d t} & =\delta_{2} r_{z} Z-q_{z} Z^{2}+\delta_{3} c_{z x} X Z+\delta_{3} c_{z y} Y Z,
\end{aligned}
$$

6 where $X, Y$ and $Z$ are the population densities of $x, y$ and $z$ respectively. For the

7 remaining parameters, we take $r$ to represent the intrinsic demographic (growth or

8 mortality) rate of each species/strain, $q$ to be the intra-species interaction

9 (competition) terms and $c$ to be the cross-species interaction terms. The strength of

10 this notation is that by selecting different values of $\delta_{1}, \delta_{2}$, and $\delta_{3}$ (see Table 2)

11 equation (4) can be made to represent classical competitive, mutualistic and predator12 prey systems.

14 We invoke a relation between the cross-species interaction terms, $c_{x z}$ and $c_{z x}$ (and

15 similarly between $c_{y z}$ and $c_{z y}$ ), such that $c_{z x}=g\left(c_{x z}\right)$ (and similarly $c_{z y}=g\left(c_{y z}\right)$ )

16 (Table 2) in order to model a wide variety of biological scenarios. These interspecific

17 relationships help define the ecological characteristics of the system, in that they, for

18 example, determine how the competition coefficients of two species depend on each

19 other. In the case of predator-prey, we use a standard linear form for $g$ representing a

20 conversion ratio, while in the other cases we can have biologically meaningful

21 positive and negative relationships or indeed no relationship between these terms. 
2 We take the $x$ strain to initially be the resident strain and $y$ to be the mutant invader.

3 In these roles, we can calculate the fitness function $s_{x}(y)$ as

$5 \quad S_{x}(y)=\delta_{1} r_{y}-q_{y} X+\delta_{3} c_{y z} Z$

6

7 Here, the fitness can be calculated directly from the dynamics in equation (4) as this

8 model consists only of a single class. First taking a trade-off to include the intrinsic

9 growth rate $r$, the invasion boundary $f_{1}$ is, from equation (5),

10

$11 \quad r_{y}=f_{1}(\cdot)=\frac{1}{\delta_{1}}\left(q_{y} X-\delta_{3} c_{y z} Z\right)$

12

13 We choose the cross-species interaction term, $c_{i z}$, to be the second parameter

14 involved in the trade-off, so $r_{i}=f\left(c_{i z}\right)$ where $i=x, y$ and $f^{\prime}>0$. Calculating the

15 curvatures of the invasion boundaries at the tip of the singular TIP gives

16

$\left.17 \quad \frac{\partial^{2} f_{1}}{\partial c_{y z}^{2}}\right|_{c_{x z}^{*}}=0 \quad$ and $\left.\left.\quad \frac{\partial^{2} f_{2}}{\partial c_{y z}^{2}}\right|_{c_{x z}^{*}}=\left(-\frac{1}{\delta_{1}}\right) \frac{2 q_{x} g^{\prime}\left(c_{x z}^{*}\right) X}{\left[q_{x} q_{z}-c_{x z}^{*} g\left(c_{x z}^{*}\right)\right.}\right]$.

19 (where equation (A.9) was used to calculate the curvature of $f_{2}$, also using the

20 equilibrium densities from equation (A.8) and the singularity condition

$\left.\left.21 f^{\prime}\right|_{x^{*}}=\partial f_{1} /\left.\partial c_{y z}\right|_{x^{*}}\right)$. The invasion boundary $f_{1}$, which stems from $s_{x}(y)$, is always

22 straight because all the parameters enter the fitness function linearly. Furthermore, the 
1 term in square brackets for the curvature of $f_{2}$ (in equation (7)) is positive due to

2 conditions imposed for the equilibria in equation (A.8) to be point stable. Thus, for

3 each interaction, using the appropriate values of $\delta$ (and the function $g\left(c_{x z}\right)$ ) shown in

4 Table 2, we can calculate the shape of the invasion boundaries at the tip of the

5 singular TIP. For competition and mutualism, these are dependent upon the relation

6 between the cross-species interaction terms, primarily on the sign of $g^{\prime}\left(c_{x z}^{*}\right)$ (for

7 predator-prey this is always negative). Results are summarised in Table 3.

9 The shapes of invasion boundaries specified in Table 3 can be used to produce

10 singular TIPs for the various model systems. Fig. 3 shows the singular TIPs for the

11 competition model for each possible sign of $g^{\prime}\left(c_{x z}^{*}\right)$. If an increase in the competitive

12 ability of one species results in decrease in that of the other species (an

13 aggressive/passive relationship) then there is the possibility of evolutionary branching

14 for trade-offs with weakly decelerating costs (Fig. 3A). If the between species

15 competition parameters are unrelated the invasion boundaries are linear and

16 superimposed (Fig. 3B). If an increase in the between species competition rate of one

17 species results in increase in the other species (aggression is countered with

18 aggression) then a Garden of Eden (ES but not CS) (ES-repellor) outcome exists for

19 weakly accelerating costs (note also that evolutionary branching is no longer possible)

20 (Fig. 3C).

22 The singular TIPs for mutualism and predator-prey set-ups, with a trade-off between $r$

23 and $c$, are similar to those for competition although the specific details are model

24 dependent (and reflect the slope and cost-benefit structure of the trade-off function).

25 For mutualism the singularity is at the top left of the TIP and for predator-prey the 
1 TIPs is the same form as figure $3 \mathrm{~A}$ but with the regions being the mirror image in the

2 (straight) $f_{1}$ line for predator evolution. For evolutionary branching to occur the

3 between species interaction term must be of the form $g^{\prime}\left(c_{x z}^{*}\right)<0$ (Table 3) reflecting

4 the fact that a benefit through the interaction for one species produces a cost for the

5 other species. If this is the case then branching can occur for trade-offs with weak

6 decelerating costs. For mutualism this requires that an increase in the benefit of the

7 mutualistic interaction for one species produces a reduction in the benefit for the other

8 species. For prey evolution it requires that a reduced predation rate produces fewer

9 predator births and for predator evolution an increased predation rate must increase

10 the loss rate of the prey.

12 When the interaction term is of the form $g^{\prime}\left(c_{x z}^{*}\right)>0$ reflecting the fact that a benefit

13 through the interaction for one species produces a benefit for the other species then

14 Garden of Eden evolutionary behaviour is possible for trade-offs with weak

15 accelerating costs (in practice these singularities act as evolutionary repellors). When

$16 g^{\prime}\left(c_{x z}^{*}\right)=0$, the cross-species interaction terms are unrelated (i.e. there is no

17 interspecific parameter dependence). Here, the two invasion boundaries are identical

18 (superimposed) and hence branching points cannot occur (see also White and Bowers

19 2005). Note also that branching requires that the evolving species exhibits

20 intraspecific competition $\left(q_{x}>0\right)$ since otherwise the invasion boundary $f_{2}$ is straight

21 and therefore superimposed on $f_{1}$ (equation (7), see also Bowers et al., 2005).

23 The remaining two trade-off possibilities are also summarised in Table 3. The trade-

24 off $q_{x}$ against $c_{x z}$ again involves the cross-species interaction term and produces 
1 identical results in terms of the shapes of invasion boundaries and evolutionary

2 outcomes to those for $r_{x}$ again $c_{x z}$. The trade-off between $r_{x}$ and $q_{x}$ produces two

3 (superimposed) straight invasion boundaries. Here, although the cross-species terms

4 can be related, they are not involved in the trade-off (and are therefore constant) and

5 hence the curvature of the invasion boundaries are equal (and zero) (Bowers et al.,

6 2005; White and Bowers 2005).

15

$$
\begin{aligned}
& \frac{d S_{x}}{d t}=r_{x}\left(S_{x}+k I_{x}\right)-q\left(S_{x}+k I_{x}\right) H-\beta_{x} S_{x}\left(I_{x}+I_{y}\right)+(\gamma+k b) I_{x}, \\
& \frac{d S_{y}}{d t}=r_{y}\left(S_{y}+k I_{y}\right)-q\left(S_{y}+k I_{y}\right) H-\beta_{y} S_{y}\left(I_{x}+I_{y}\right)+(\gamma+k b) I_{y},
\end{aligned}
$$

$$
\frac{d I_{x}}{d t}=\beta_{x} S_{x}\left(I_{x}+I_{y}\right)-(\alpha+\gamma+b) I_{x} \text {, }
$$$$
\frac{d I_{y}}{d t}=\beta_{y} S_{y}\left(I_{x}+I_{y}\right)-(\alpha+\gamma+b) I_{y},
$$

\section{Multi-species, multi-class}

The final section looks at host evolution in a host-parasite model consisting of May, 1981), susceptible and infected classes (with no immune class) where the parasite is therefore modelled implicitly via the infected class of the host. Again taking two strains of host, defined as $x$ and $y$, we can define the dynamics as follows (based on Anderson and

\section{6}

17

where $H=S_{x}+S_{y}+I_{x}+I_{y}$. The parameter $r$ represents the intrinsic growth rate of the host (i.e. births-deaths) and $k$ represents the reduction, due to infection, in the birth rate from infected hosts. For the purposes of this study, we restrict this parameter to either 0 , for total loss of reproduction from infecteds (births only from 
1 susceptibles), or 1 , for no decrease in reproduction due to infection. The parameter $q$

2 represents competition between hosts, $\beta$ the infection rate, $\gamma$ the recovery rate, $\alpha$

3 the parasite induced death rate and $b$ the natural death rate where the term $+b k I$ in

4 the susceptible class compensates for the deaths included in $r k I$.

6 Due to the added complexity of this model, being both multi-species and multi-class,

7 we present the analysis only for a trade-off between the intrinsic growth rate of the 8 host, $r$, and the infection rate, $\beta$.

9

10 As with the two previous examples, for the purposes of calculating the fitness

11 function we take the strains $x$ and $y$ to assume the roles of the established resident

12 and mutant invader respectively. In these roles, the fitness function, $s_{x}(y)$, takes the

13 form

14

$15 S_{x}(y)=\frac{1}{b+\beta_{y} I_{x}}\left[r_{y}-q\left(S_{x}+I_{x}\right)+\frac{\beta_{y} I_{x}}{\alpha+\gamma+b}\left(r_{y} k-q k\left(S_{x}+I_{x}\right)-\alpha-(1-k) b\right)\right]$,

16

17 (see equations (A.11)-(A.14) for working). We focus on a trade-off of the form

$18 r=f(\beta)$. Finding the invasion boundary $f_{1}$, from $s_{x}(y)$ above, gives

19

20

$$
r_{y}=f_{1}\left(\beta_{y}\right)=q\left(S_{x}+I_{x}\right)+\frac{\beta_{y} I_{x}(\alpha+(1-k) b)}{\alpha+\gamma+b+k \beta_{y} I_{x}}
$$


1 We now go back to the condition we imposed on $k$, such that we restrict it to the

2 value of 0 or 1 (depending on whether infecteds can reproduce or not), and take these

3 two cases in turn.

4

5 Starting with $k=0$, which relates to the case when there is total loss of reproduction

6 when the host is infected, the curvatures of the invasion boundaries at the tip of the

7 singular TIP are

8

$\left.9 \quad \frac{\partial^{2} f_{1}}{\partial \beta_{y}^{2}}\right|_{x^{*}}=0,\left.\quad \frac{\partial^{2} f_{2}}{\partial \beta_{y}^{2}}\right|_{x^{*}}=\frac{2 q S_{x}(\alpha+b)}{\beta^{2}\left(\alpha+b+q S_{x}\right)}$.

10

11 The main conclusion is that the invasion boundary $f_{1}$ is straight, whereas $f_{2}$ has

12 positive curvature implying that it curves upwards near the tip of the singular TIP. It

13 follows that the singular TIP will take an identical form to that for competition, in Fig.

$143 \mathrm{~A}$; hence branching points occur for weakly decelerating trade-offs.

15

16 For the second case, we assume there is no reduction in the reproduction rate of the

17 hosts due to infection, hence $k=1$, and again take a trade-off between the intrinsic

18 growth rate $r$ and the infection rate $\beta$. Calculating the curvatures of the invasion

19 boundaries at the tip of the singular TIP gives

20

$\left.21 \frac{\partial^{2} f_{1}}{\partial \beta_{y}^{2}}\right|_{x^{*}}=-\frac{2 \alpha S_{x} I_{x}^{2}}{\beta^{2}\left(S_{x}+I_{x}\right)^{3}},\left.\quad \frac{\partial^{2} f_{2}}{\partial \beta_{y}^{2}}\right|_{x^{*}}=\frac{2 \alpha S_{x}}{\beta^{2}\left(S_{x}+I_{x}\right)^{3}}\left[-I_{x}^{2}+\theta\right]$, 
1 where $\theta$ is as in equation (A.15). The invasion boundary $f_{1}$ is no longer straight and

2 now curves downwards (Fig. 4). The implication of this is that the evolutionary

3 singularity can be a branching point with either a weakly decelerating trade-off, or a

4 weakly accelerating trade-off (Fig. 4A) or alternatively with a moderately accelerating

5 trade-off (Fig. 4B). This depends upon the relative curvatures of $f_{1}$ and $f_{2}$. It is

6 important to note that as with all the examples we have seen, evolutionary attractors

7 always occur for strongly accelerating trade-offs and evolutionary repellors for

$8 \quad$ strongly decelerating trade-offs.

10 The results above emphasise the different configurations of TIPs and therefore

11 evolutionary outcome that can occur. It is interesting to note from equation (12)

12 however that, the sign of the curvature of $f_{2}$ is also not fixed. Indeed, the curvature

13 can be either greater than or less than that of $f_{1}$. Hence these results for when the

14 infecteds can and can not reproduce emphasise the complex evolutionary outcomes

15 that can be visualised swiftly with the geometric approach.

16

17

18

19

20

21

22

23

\section{Discussion}

The feedback between ecological and evolutionary processes is crucial to understand how ecological interactions generate natural selection and how evolutionary change further modifies the ecological interactions (MacArthur, 1972; Roughgarden, 1979; Bulmer, 1994). By applying a geometric approach we have developed a theory for how different trade-off shapes affect evolutionary outcomes in a number of classical ecological scenarios. The work clearly demonstrates the importance that the shape of 
1 the trade-off curve plays. Whether costs are accelerating or decelerating and,

2 furthermore, whether the curvature of these trade-offs is relatively weak or strong (in

3 relation to the invasion boundaries) are key determinants of the evolutionary outcome

4 in all of our examples. The outcomes are also fundamentally dependent on the

5 particular ecological scenario that is being considered. The ecological characteristics

6 of our specific models may change the curvature of both invasion boundaries and

7 therefore radically change the evolutionary outcome but the approach we have taken

8 allows a clear separation of the effects due to the ecological feedbacks and those that

9 result from the shape of the trade-off

10

11 We can define four fundamental forms of TIPs. First, the two invasion boundaries can

12 be linear and therefore superimposed, a type I TIP (see Fig. 2A, 3B). This implies that

13 trade-offs with accelerating costs always produce a CSS, or attractor, while

14 decelerating costs produce a repellor. In type II TIPs, both boundaries curve at the

15 same rate and remain superimposed (see Fig. 2B). Since the curvature of the invasion

16 boundaries can be either positive or negative, the direction and strength of the

17 curvature is important. We find that weakly decelerating costs in addition to

18 accelerating costs can lead to a CSS or in contrast weakly accelerating costs in

19 addition to all decelerating costs can lead to a repellor. It should be noted that

20 whenever the two invasion boundaries are superimposed in our models an

21 optimisation principle exists (Mylius \& Diekmann, 1995). This implies that a CSS

22 will produce an intermediate trait value whereas a repellor will always lead to

23 maximisation or minimisation of the trait value. In type III TIPs, one invasion

24 boundary curves while the other remains linear leading inevitably to separation (see

25 Fig. 3A, 3C). We commonly find that the $f_{2}$ boundary curves such that weakly 
1 decelerating costs lead to polymorphisms through evolutionary branching, while

2 strong decelerating costs lead to repellors and accelerating costs lead to CSS attractors

3 (although note the alternative configuration of Fig. 3C). Finally in type IV TIP, both

4 boundaries curve but separate (see Fig. 4A, 4B). Here, we additionally find that

5 weakly accelerating as well as weakly decelerating costs may lead to branching..

6

7 Our work emphasises the importance of the strength as well as the nature of the trade-

8 off cost structure. Strong non-linearity always produce CSS or repellor dynamics (for

9 accelerating or decelerating cost structures respectively) whereas weak non-linearity

10 (and linear trade-offs) can produce the full range of evolutionary behaviour depending

11 on the configuration and type of TIP. It is important to recognise that it is the

12 ecological feedbacks in the system that define the type of TIP that occurs and

13 therefore whether, for example, optimalities or disruptive selection can occur in a

14 particular scenario.

15

16 A key aim of our work is to use our models to gain insight into which ecological

17 characteristics lead to each of our fundamental TIPs. To do this we have observed

18 three criteria:

19 1. Criterion A: the evolving parameters must appear in different classes or one

20 must be repeated, appearing in more than one class/species.

212 2. Criterion B: the evolving parameters must be characteristics of different

22 classes or one must be a characteristic of more than one class.

23 3. Criterion $\mathrm{C}$ : there must exist two density dependent per capita rates, each of 24 which must be dependent on different densities. 
1 Satisfying these three criteria in different combinations lead to one of four distinct

2 fundamental TIPs (Table 4).

4 These criteria hold for the models in this study and allow the range of possible

5 evolutionary behaviour to be predicted by inspection of the model structure and a

6 knowledge of the evolving parameters. The first, criterion A, is that the two evolving

7 parameters must either appear in different classes or one must be repeated, appearing

8 in more than one class/species. Hence, because the two evolving parameters are

9 linked, a change in these parameters will directly affect the dynamics of more than

10 one class/species. Without this, we always get two linear boundaries and therefore a

11 'simple' optimality (type I). It should be noted that this optimisation can occur in a 12 range of multi-species or multi-class ecological interactions for particular trade-offs.

13 If, in addition to satisfying criterion A, criterion B is satisfied such that the parameters

14 are also characteristics of different classes then the invasion boundaries can curve

15 with equal curvature (type II). For instance in the single species multi-class model

16 (equation 1) the juvenile and mature death rates ( $b$ and $e$ respectively) appear in

17 different classes and are also characteristics of their different respective classes

18 leading to a type II TIP. Contrast this with a trade-off between mature birth and death

19 rates ( $a$ and $e$ ) which are characteristics of only the mature individuals or birth rate

20 and juvenile death rate ( $a$ and $b$ ) which appear in the juvenile class only and therefore

21 lead to type I TIPs.

23 To move from type I or type II TIPs, where the invasion boundaries are

24 superimposed, to type III or type IV TIPs, where they separate, requires criterion C.

25 Whereas criteria A and B focussed on the choice of evolving parameters, criterion C 
1 depends only upon the form of the model structure. This criterion is that the dynamics

2 of the evolving species must contain at least two density dependent per capita rates

3 each of which should be dependent on different densities. Density-dependent rates

4 occur in non-linear model terms. For instance, if we consider prey evolution in a

5 predator-prey system (equation 4) the intraspecific competition term is $-q X^{2}$ and

6 therefore the rate of intraspecific competition is $q X$; in the same model the predation

7 term is $-c X Z$ and therefore the rate of predation is $c Z$. Both the rates are therefore

8 density dependent and are associated with different densities ( $X$ and $Z$ respectively). If

9 criterion $\mathrm{C}$ is satisfied the invasion boundaries can separate which allows for the

10 possibility of evolutionary branching. This criterion is analogous to the dimensionality

11 of the fitness environment (Rueffler et al., 2006) however this version has the added

12 benefit that it can be determined directly from inspection of the model without the

13 need for calculating the fitness and hence is easier and quicker to use. Whether we

14 observe type III or type IV TIPs depends on which of the other criteria are satisfied

15 (Table 4) and is best highlighted using the host-parasite model (equation 8). If we

16 consider host evolution, in the presence of a castrating parasite $(k=0)$, then there are

17 two density dependent rates associated with different densities (the infection rate

18 associated with the density of infecteds, $\beta I$, and the intraspecific competition rate

19 associated with total host density, $q(S+I))$ satisfying criterion C. If we consider a

20 trade-off between $r$ and $\beta$ then criterion $\mathrm{A}$ is satisfied (as $\beta$ appears in both classes)

21 but criterion B is not (as both $r$ and $\beta$ are characteristics of the susceptible class $S$ ).

22 We therefore observe type III TIPs (equivalent to that in Fig. 3A). However when the

23 infected hosts can also reproduce, $k=1$, criterion B is now satisfied (as $r$ is now also

24 a characteristic of the infecteds $I$ ) and so type IV TIPs are observed (Fig. 4). Our

25 three criteria also explain why all the single class, multi-species systems, as described 
1 by equation (4), can only produce type I or type III TIPs. Criterion C may be satisfied

2 due to the existence of a density dependent self-regulation rate and the between

3 species interaction rate, however criterion B can never be satisfied because in single

4 class models all evolving parameters are characteristics of a single class. Thus, we can

5 only observe type I or type III TIPs; which of these occur depends on whether the

6 evolving parameters contain the between species interaction parameter (which would

7 allow criterion A to be satisfied).

9 Although the above insights have only been demonstrated for our models we

10 hypothesise that they will hold in all ecological scenarios that satisfy our constraints.

11 To re-iterate, we restricted these to Lotka-Volterra, continuous time models, i.e.

12 where the evolving parameters appear linearly in the dynamics and the densities are of

13 order 1 or 2 . It would be interesting to examine how our conclusions apply to more

14 complex scenarios where, for example, there is a non-linear Holling type II predator

15 functional response. However, by initially limiting our work to classical models, we

16 have provided a baseline theory that will at least inform this family of ecological

17 scenarios. Our criteria may therefore allow the rapid evolutionary classification of

18 different trade-offs in a variety of ecological scenarios.

20 The importance of trade-off shapes in life-history evolution has long been recognised

21 (Levins, 1962). Many of the results from classic life history theory use optimisation

22 techniques and tend to predict attractors for accelerating trade-offs and repellors for

23 decelerating ones (Stearns, 1992; Roff, 2002). We have shown that this is the case

24 (type I TIP) in our single species system for a number of important trade-offs between

25 life-history components including birth rate verses mature or juvenile death rate. 
1 However, there are a number of trade-offs in this model where this result is not found.

2 Strongly accelerating and strongly decelerating trade-offs always lead to the

3 'classical' results, but when the curvatures are relatively weak they no longer hold

4 (and type II TIPs are possible). We note here that curved invasion boundaries have

5 been reported in life history examples under adaptation to temporally varying

6 environments (Levins, 1962) and for trade-offs that link multiplicative fitness

7 components (Schaffer, 1974). Our study has shown similar results for a trade-off

8 between two life-history components and highlights the importance of trade-off

9 shapes and the strength of the curvature for a straightforward model framework and

10 trade-off.

12 For evolutionary branching to occur, not only do criteria A and $\mathrm{C}$ need to be satisfied,

13 allowing the invasion boundaries to separate (criterion B is not necessarily needed),

14 but the interaction term between species needs to be antagonistic. Such relationships

15 are obvious in host-parasite and predator-prey systems, where for example the

16 evolution of predator ability clearly affects the prey's ability to avoid predation. For

17 branching to occur in our competitive and mutualistic systems the interaction also has

18 to be antagonistic in the sense that an improvement in one species leads to a reduction

19 in the competitive or mutualistic ability of the other. Clearly not all competitive or

20 mutualistic interactions will be of this type, but one example where we might expect

21 branching is when competition occurs for the same limiting resource. In this case, the

22 uptake of the resource in one competitor will improve its competitive ability and

23 reduce that of the other species (Tompkins et al., 2003). If the competition (or

24 mutualism) coefficients of the two species are independent, or alternatively one

25 competitor can evolve to improve its competitive ability without affecting that of its 
1 competitor, there is no possibility of branching. We would therefore predict that there

2 may be more polymorphism and variation in competitive ability when the competition

3 is for a limiting resource.

5 In the majority of our examples where branching can occur, only one of the invasion

6 boundaries curves with the other one being linear (predator-prey, competition and

7 mutualistic interactions as well as resistance through avoidance when infected

8 individuals do not reproduce, including those parasites that affect immature stages and

9 stop maturation). In these type III TIPs, strongly decelerating costs produce repellors

10 while any degree of acceleration in the costs leads to a CSS. This behaviour has been

11 found in other studies where there is an antagonistic relationship between species.

12 This includes the evolution of size specific predation on prey life-history (Day et al.,

13 2002) and frequency-dependent selection of consumer types when modelled as

14 evolving specialisation or generalisation on two prey types (Rueffler et al., 2006)

15 (here the underlying ecological scenario is analogous to a predator-prey system). Our

16 study, however, has shown a broader range of behaviour for systems with antagonistic

17 interactions. There are a wide range of castrating parasites in nature including ones

18 that affect immature stages and stop maturation, and for certain trade-offs, for

19 example one between the growth rate of a host and the infection rate, these will

20 produce type III TIPs characteristic of the above antagonistic interactions. However a

21 key ecological characteristic of parasites along with the potential for recovery is that

22 infected individuals do reproduce even if this is at a reduced rate (Boots, 2004; Boots

23 and Norman, 2000). Once reproduction from infecteds occurs, both invasion

24 boundaries may curve, which means that weakly curved cost structures whether

25 decelerating or accelerating may lead to evolutionary branching in resistance (type IV 
1 TIP). Cost structures will often depend on specific physiological mechanisms (Boots

2 and Haraguchi, 1999), although intuitively, accelerating costs may be relatively

3 common since gains may tend to saturate quicker than costs. One of the key

4 predictions of our work is that these accelerating costs will lead to a CSS in most

5 antagonistic interactions, but may lead to branching in resistance to parasites where

6 there is either recovery or reproduction by infected individuals. Another example

7 where accelerating costs can induce evolutionary branching is for a multiplicative

8 trade-off in survival in different habitats in which the carrying capacity of each habitat

9 is also dependent on the phenotype (de Mazancourt and Dieckmann, 2004).

11 Our key result is that shapes of trade-offs matter and that different shapes may have 12 different implications in different scenarios (Levins, 1962, 1968; de Mazancourt and

13 Dieckmann, 2004; Rueffler et al., 2004; Bowers et al., 2005).A broad perspective has

14 been facilitated by the relatively easy way in which the implications of trade-off

15 shapes can be understood using our graphical approach, TIPs. Furthermore, we have

16 defined four fundamental TIPs and are able to identify the ecological characteristics

17 that lead to each of them solely from the dynamics and choice of trade-off. In addition

18 our work particularly emphasises the importance of the strength of the costs, not only

19 in causing disruptive selection leading to branching but also when an optimality

20 principle holds where the invasion boundaries curve. It is clear that trade-off shapes

21 are important and as a consequence, when building evolutionary models, assumptions

22 about the relationships between traits need to be carefully considered. The outcome

23 will tend to depend heavily on these assumptions. We would therefore argue that this

24 approach may prove useful whenever the implications of trade-off shapes are

25 considered in evolutionary models. Clearly measuring the shape of trade-offs in 
1 nature is a major challenge, but we have shown that evolutionary outcomes are

2 crucially dependent on them. There is therefore the pressing need for empirical studies

3 that examine these relationships. Given the controversy surrounding the likelihood of

4 branching in nature (Butlin and Tregenza, 1997) only by measuring these cost

5 structures can we understand how relevant these processes have been in shaping

6 natural communities. It is interesting to note that although many of our scenarios can

7 lead to branching, it is often only found for particular (weak) curvatures. The question

8 of how common branching is likely to be in nature is therefore likely to depend in part

9 on how often key trade-off relationships have these shapes.

10

11

12 


\section{References}

2

3 Abrams, P., 2001. Modelling the adaptive dynamics of traits in inter and

4 intraspecific interactions: An assessment of three models. Ecol. Lett. 4, 166-175.

5

6 Anderson, R.M., May, R.M., 1981. The population dynamics of microparasites and

7 their invertebrate hosts. Phil. Trans. R. Soc. Lond. 291, 451-524.

8

9 Boots, M., 2004. Modelling insect diseases as functional predators. Phys. Ent. 29, $10 \quad 237-239$.

11

12 Boots, M., Bowers, R.G., 1999. Three mechanisms of host resistance to

13 microparasites - avoidance, recovery and survival - show different evolutionary

14 dynamics. J.Theor. Biol. 201, 13-23.

15

16 Boots, M., Haraguchi, Y., 1999. The evolution of costly resistance in host-parasite 17 systems. Am. Nat. 153, 359-370.

18

19 Boots, M., Norman, R., 2000. Sublethal infection and the population dynamics of

20 host-microparasite interactions. J. Anim. Ecol. 69, 517-524.

21

22 Boots, M., Bowers, R.G., 2004. The evolution of resistance through costly acquired

23 immunity. Proc. R. Soc. Lond. B 271, 715-723.

24 
1 Bowers, R. G., White, A., Boots, M., Geritz, S.A.H., and Kisdi, E., 2003.

2 Evolutionary branching/speciation: contrasting results from systems with explicit or

3 emergent carrying capacities. Evol. Ecol. Res.. 5, 883-891.

5 Bowers, R. G., Hoyle, A., White, A., Boots, M., 2005. The geometric theory of

6 adaptive evolution: trade-off and invasion plots. J. Theor. Biol. 233, 363-377.

Bulmer, M., 1994. Theoretical evolutionary ecology. Sinauer Associates Sunderland,

9 Massachusetts.

10

11 Butlin, R. K., Tregenza, T., 1997. Evolutionary biology: Is speciation no accident?

$12 \quad$ Nature 387, 551-552.

13

14 Day, T., Abrams, P.A., Chase, J., 2002. The role of size-specific predation in the 15 evolution and diversification of prey life histories. Evolution 56, 877-887

16

17 De Mazancourt, C., Dieckmann, U., 2004. Trade-off geometries and frequency18 dependent selection. Am. Nat. 164, 765-778.

Egas M., Dieckmann, U. Sabelis, M.W. 2004. Evolution restricts the coexistence of 21 specialists and generalists - the role of trade-off structure. Am. Nat. 163, 518-531.

23 Geritz, S. A. H., Kisdi, E, Meszena, G., Metz, J.A.J., 1998. Evolutionary singular 24 strategies and the adaptive growth and branching of the evolutionary tree. Evol. Ecol. $25 \quad 12,35-57$. 
2 Heino, M., Metz, J.A.J., Kaitala, V., 1997. Evolution of mixed maturation strategies

3 in semelparous life-histories: the crucial role of dimensionality of feedback

4 environment. Phil. Trans. R. Soc. Lond. B 352, 1647-1655

5

6 Kisdi, E., 2001. Evolutionary branching under asymmetric competition. J. Theor.

7 Biol. 197, 149-162.

8

9 Kirchner, J.W., Roy, B.A., 2000. Evolutionary implications of host-pathogen

10 specificity: the fitness consequences of host lift history traits. Evol. Ecol. 14, 665-692.

11

12 Levins, R., 1962. Theory of fitness in heterogeneous environment. 1. the fitness set 13 and the adaptive function. Am. Nat. 96, 361-373.

14

15 Levins, R., 1968. Evolution in changing environments. Princeton University Press, 16 Princeton.

17

18 MacArthur, R. H., 1972. Geographical Ecology. Harper and Row, New York.

19

20 Maynard Smith, J., 1982. Evolution and the theory of games. Cambridge University

21 Press, Cambridge.

22

23 Metz, J. A. J., Nisbet, R.M., Geritz, S.A.H., 1992. How should we define 'fitness' for 24 general ecological scenarios? Trends Ecol. and Evol. 7, 198-202. 
1 Metz, J. A. J., Geritz, S.A.H., Meszena, G., Jacobs, F.J.A., Van Heerwaarden, J.S.,

2 1996. Adaptive dynamics: a geometric study of the consequences of nearly faithful

3 reproduction. Pages 183-231 in S. J. van Strein and S. M. Verduyn Lunel, eds.

4 Stochastic and spatial structures of dynamical systems. Proceedings of the Royal

5 Dutch Academy of Science (Verhandelingen KNAW), North Holland, Amsterdam.

6

7 Miller, M. R., White, A., Boots, M., 2005. The evolution of host resistance: tolerance

8 versus control. J. Theor. Biol. 236, 198-207.

9

10 Mylius, S.D., Diekmann, O., 1995. On evolutionary stable life histories, optimisation

11 and the need to be specific about density-dependence. Oikos. 74, 218-224.

12

13 Roff, D. A., 2002. Life history evolution. Sinauer, Sunderland, MA.

14

15 Roughgarden, J., 1979. Competition and theory in community ecology. Am. Nat. 5, $16 \quad 583-601$.

17

18 Rueffler, C., Van Dooren, T.J.M., Metz, J.A.J., 2004. Adaptive walks on changing

19 landscapes: Levin's approach. Theor. Pop. Biol. 65, 165-178.

20

21 Rueffler, C., Van Dooren, T.J.M., Metz, J.A.J., 2006. The Evolution of Resource

22 Specialization through Frequency-Dependent and Frequency-Independent

23 Mechanisms. Am. Nat. 167, pp. 81-93.

24 
1 Schaffer, W. M., 1974. Optimal reproductive effort in fluctuating environments. Am.

$2 \quad$ Nat. 108, 783-790.

3

4 Stearns, S. C., 1992 The evolution of life histories. Oxford University Press, Oxford. 5

6 Tompkins, D., White, A., Boots, M., 2003. Ecological replacement of native red

7 squirrels by invasive greys driven by disease. Ecol. Lett. 6, 189-196

8

9 White, A., Bowers, R.G., 2005. Adaptive dynamics of Lotka-Volterra systems with

10 trade-offs: the role of intraspecific parameter dependence in branching. Math. Bios.

$11 \quad 197,101-117$.

12 


\section{Appendix}

3 Trade-off and invasion plots. - A detailed description of the use of trade-off and

4 invasion plots (TIPs) to determine evolutionary behaviour has been given elsewhere

5 (Bowers et al., 2005). Here we will give a brief outline of TIPs and present some of

6 the results/conditions for determining the evolutionary behaviour of a system. This

7 section provides the mathematical underpinning for the work carried out in this paper.

9 Trade-off and invasion plots are a geometrical approach that makes the role that

10 different trade-off shapes play easy to visualise. Although underpinned

11 mathematically, TIPs are essentially geometrically based, making them a very "user-

12 friendly" method for studying evolution. A TIP is a plot between two (competing)

13 strains of a species, labelled $x$ and $y$ say. One of these, $x$, is taken to be fixed while

14 the second, $y$, is allowed to vary. The axes of a TIP are the two evolving parameters

15 of the $y$ strain, $y_{1}$ and $y_{2}$ (only two parameters are taken to vary). The co-ordinates

$16 x_{1}$ and $x_{2}$ of the fixed strain $x$ define the corner or tip of a TIP. To emphasise this

17 notation in a biological context consider the evolution of a prey species. Here $x_{1}$ and

$18 y_{1}$ may represent the prey's ability to avoid predation for two different prey strains

19 while $x_{2}$ and $y_{2}$ may represent the prey birth rate. An example of a TIP can be seen

20 in Fig. 1.

22 Two of the three curves on a TIP are the invasion boundaries, denoted as $f_{1}$ and $f_{2}$.

23 These curves divide a TIP into regions where the varying strain $y$ can and cannot

24 invade the fixed strain $x$ (either side of $f_{1}$ ) and where the fixed strain $x$ can and 
1 cannot invade the varying strain $y$ (either side of $f_{2}$ ). If we denote the fitness of a

2 rare mutant $y$ with resident $x$ as $s_{x}(y)$ (Geritz et al., 1998) then on the curve $f_{1}$ we

3 have that $s_{x}(y)$ is zero and therefore $f_{1}$ partitions the TIP into regions where the

4 fitness of strain $y$ is positive and negative. The roles of $x$ and $y$ are reversed when

5 considering the curve $f_{2}$ along which $s_{y}(x)$ is zero. Both of these invasion

6 boundaries pass through the tip of a TIP (a 'neutral' point at which both strains are the

7 same) at which they are tangential. The third curve on a TIP is the trade-off curve,

8 denoted as $f$; this links the two evolving parameters of each strain. As all feasible

9 pairs of parameters (and hence strains) lie on this curve, $f$ too must pass through the

10 tip of a TIP, but not usually tangentially to the invasion boundaries. Therefore the side

11 of the invasion boundaries in which the trade-off enters a TIP determines whether

12 each strain can invade the other (when initially rare). Generically, the trade-off curve

13 crosses the invasion boundaries at $x$ and so the regular behaviour is invadability of $x$

14 by $y$ for $y<x$ (say) and non-invadability for $y>x$ - with the opposite results for the

15 invadability of $y$ by $x$ (see Bowers et al., 2005).

17 For certain TIPs corresponding to particular values $x^{*}$ of $x$, the trade-off curve can

18 become tangential to the invasion boundaries at the tip of a TIP (i.e. where

$19 y=x=x^{*}$ ); then we will have singular behaviour - no change in invadability as we

20 move through $x^{*}$ These values of $x$ are evolutionary singularities, with the

21 corresponding TIPs being singular TIPs (Fig. 1). It is from these singular TIPs that the

22 evolutionary behaviour of a system is determined. (If a singular point does not exist,

23 then invadability will prefer either always higher or always lower values of $x$. If more

24 than one singular point exists then a separate TIP must be considered at each singular 
1 point.) Comparing TIPs with $x<x^{*}$ and $x>x^{*}$, generically the trade-off curve

2 crosses the invasion boundaries from above in one case and below in the other - the

3 invadability properties change at $x^{*}$. Due to the coincidence and mutual tangential

4 property of the three curves at the tip of a singular TIP, the region in which the trade-

5 off curve enters (and hence the evolutionary behaviour) is determined solely by the

6 curvatures of the three curves; or more specifically, the curvature of the trade-off in

7 relation to those of the invasion boundaries at the evolutionary singularity (as in

8 standard theory mutations are assumed to be small). The two significant relations are

9 between the trade-off and $f_{1}$ for evolutionary stability ES (the criteria for the classical

10 evolutionarily stable strategy ESS) and between the trade-off and the mean curvature

11 of both $f_{1}$ and $f_{2}$ for convergent stability CS. These can be written

13

$$
\begin{aligned}
\mathrm{ES} & \Leftrightarrow \lambda_{1} f^{\prime \prime}\left(x^{*}\right)<\left.\lambda_{1} \frac{\partial^{2} f_{1}}{\partial y_{1}^{2}}\right|_{x^{*}} \\
\mathrm{CS} & \left.\Leftrightarrow \lambda_{1} f^{\prime \prime}\left(x^{*}\right)<\frac{\lambda_{1}}{2}\left|\frac{\partial^{2} f_{1}}{\partial y_{1}^{2}}\right|_{x^{*}}+\left.\frac{\partial^{2} f_{2}}{\partial y_{1}^{2}}\right|_{x^{*}}\right)
\end{aligned}
$$

15 where $\lambda_{1}=\operatorname{sig} n\left(\left.\frac{\partial s_{x}(y)}{\partial y_{2}}\right|_{x^{*}}\right)$. Here $\lambda_{1}$ concerns how the fitness varies as we move

16 vertically up a TIP (i.e. as we vary the parameter on the vertical axis). This determines

17 whether evolutionary attractors occur towards the lower part of a singular TIP (if

$18 \lambda_{1}>0$ ) or the upper part (if $\lambda_{1}<0$ ). To make this more concrete we observe that, for

$19 \lambda_{1}>0$, the singularity is ES when the trade-off curve is locally below the $f_{1}$

20 boundary and CS when it is locally below the mean of the two invasion boundaries

21 (this is the situation in the figures presented in this study but see Table 5 and Bowers 
1 et al. (2005) for the $\lambda_{1}<0$ alternative). Combinations of these properties allow the

2 evolutionary behaviour of the system to be determined. The possible types of

3 singularity are evolutionary attractors or CSS (continuously stable strategy) (ES and

4 CS), evolutionary branching point (CS but not an ES), 'Garden of Eden' point or ES-

5 repellor (ES but not CS) and evolutionary repellor (neither ES nor CS).

7 We close this appendix with two points of clarification on how we draw the TIPs.

8 First, in constructing TIPs we employ biological parameters directly from models

9 performing no transformations on them. Hence the point $x$ may either be in the top

10 right corner (because $f^{\prime}>0$ ) or the top left (because $f^{\prime}<0$ ). This is exemplified by

11 Fig. 2; in A as the death rate of juveniles increases so does the birth rate; in B as the

12 maturation rate increases the birth rate falls. Second, we display TIPs only for one

13 side of the strategy $x$ : globally every $x, y$ pair is covered exactly once by this

14 procedure, furthermore the geometry of the TIP just above $x$ is determined when that

15 just below $x$ is known and so the evolutionary behaviour is entirely determined by the

16 latter.

17

18 Analysis of the models. - Starting with the single species, stage-structured model,

19 before any mutations occur, i.e. with only a single (resident) strain $x$, the population

20 equilibrium densities are

21

22

$$
X_{1}=\frac{e_{x}\left(a_{x} m_{x}-b_{x} e_{x}-m_{x} e_{x}\right)}{q_{x} m_{x}\left(m_{x}+e_{x}\right)}, \quad X_{2}=\frac{a_{x} m_{x}-b_{x} e_{x}-m_{x} e_{x}}{q_{x}\left(m_{x}+e_{x}\right)}
$$


1 Our analysis (for all models) is conducted subject to the condition that all feasibility

2 and stability conditions are satisfied. In order for us to calculate the fitness function

$3 s_{x}(y)$, where the $x$ strain is the existing resident and $y$ the invading mutant, we take

4 the mutant individuals to be in the juvenile stage for an average time $T_{1}$ and in the

5 mature stage for an average time $T_{2}$. The first of these times, $T_{1}$, is given by

6

$7 \quad T_{1}=\frac{1}{b_{y}+m_{y}}$

8

9 as the only way of leaving the juvenile stage is through death, which occurs at a rate

$10 b_{y}$, or through maturation, occurring at a rate $m_{y}$. The average time an invader spends

11 in the mature stage, $T_{2}$, is found from the equality $b_{y} T_{1}+e_{y} T_{2}=1$. This stems from the

12 fact that an invading individual can only leave the system through death either as a

13 juvenile or as a mature; this exhausts all possibilities giving a total probability of 1.

14 Solving this for $T_{2}$ gives

15

$16 \quad T_{2}=\frac{m_{y}}{e_{y}\left(b_{y}+m_{y}\right)}$.

18 The rates of growth of the mutant population during these times are given by $\rho_{i}$

$19 \quad(i=1, \ldots, n$; here $n=2)$. These rates are calculated by

20

$21 \quad \rho_{i}=\operatorname{Lim}_{Y_{i} \rightarrow 0}\left(\left.\frac{1}{Y_{i}} \sum_{j=1}^{n} \frac{d Y_{j}}{d t}\right|_{Y_{k}=0}\right) \quad$ where $k=1, \ldots, n$ and $k \neq i$. 
2 For our juvenile and mature stages, this gives

$\rho_{1}=-b_{y}$,
$\rho_{2}=a_{y}-q_{y}\left(X_{1}+X_{2}\right)-e_{y}$,

5

6 respectively. Combining these in the form $s_{x}(y)=\rho_{1} T_{1}+\rho_{2} T_{2}$ gives

$8 \quad s_{x}(y)=\frac{1}{b_{y}+m_{y}}\left(-b_{y}+\frac{m_{y}}{e_{y}}\left(a_{y}-q_{y}\left(X_{1}+X_{2}\right)-e_{y}\right)\right)$,

9

10 as seen in equation (2). Using the equilibrium densities of the juveniles and matures,

11 in equation (A.1), the fitness can be re-arranged into the form

12

$13 s_{x}(y)=\frac{q_{y} m_{y}}{e_{y}\left(b_{y}+m_{y}\right)}\left[\left(\frac{a_{y}-e_{y}}{q_{y}}-\frac{b_{y} e_{y}}{q_{y} m_{y}}\right)-\left(\frac{a_{x}-e_{x}}{q_{x}}-\frac{b_{x} e_{x}}{q_{x} m_{x}}\right)\right]$.

14

15 Moving onto the multi-species, single class model, again initially taking the $x$ strain to be existing alone with species $z$ at equilibrium densities

17

$18 \quad X=\frac{\delta_{1} r_{x} q_{z}+\delta_{2} \delta_{3} r_{z} c_{x z}}{q_{x} q_{z}-c_{x z} g\left(c_{x z}\right)} \quad \mathrm{Z}=\frac{\delta_{2} r_{z} q_{x}+\delta_{1} \delta_{3} r_{x} g\left(c_{x z}\right)}{q_{x} q_{z}-c_{x z} g\left(c_{x z}\right)}$.

19

20 In order to calculate the curvature of the second invasion boundary, at the tip of the

21 singular TIP, we use the result 
$\left.2 \quad \frac{\partial^{2} f_{2}}{\partial c_{y z}^{2}}\right|_{x^{*}}=\left.\frac{\partial^{2} f_{1}}{\partial c_{y z}^{2}}\right|_{x^{*}}+\left.2 \frac{\partial^{2} f_{1}}{\partial c_{x z} \partial c_{y z}}\right|_{x^{*}}$,

3

4 found by Bowers et al. (2005).

5

6 Finally, moving onto our multi-species, multi-class model, we again assume that

$7 \quad$ initially the $x$ strain exists alone. In this scenario, the equilibrium densities for this

$8 \quad$ SIS model are

9

$$
S_{x}=\frac{\alpha_{x}+\gamma+b}{\beta_{x}},
$$

$10 \quad k=0 \Rightarrow I_{x}=\frac{S_{x}\left(r_{x}-q S_{x}\right)}{\alpha+b+q S_{x}}$,

$k=1 \Rightarrow I_{x}=\frac{r_{x}-\alpha-2 q S_{x}+\sqrt{\left(r_{x}-\alpha\right)^{2}+2 q \alpha S_{x}}}{2 q}$,

12 where we have two possible equilibrium densities for the infecteds depending upon

13 whether they can produce (susceptible) offspring at an identical rate to the

14 susceptibles or not at all. As this is a two class model, we again need to calculate the

15 rates of growth of the host population while the invader is in each class, $\rho_{S}$

16 (susceptible) and $\rho_{I}$ (infected), and also the average time a mutant individual spends

17 in each class, $T_{S}$ (susceptible) and $T_{I}$ (infected). Starting with the times, as the only

18 way of leaving the susceptible class is through (natural) death or infection, we can

19 write down the average time an invader spends in this class as 


$$
1 \quad T_{S}=\frac{1}{b+\beta_{y} I_{x}} .
$$

2

3 The average time an invader spends in the infected class is derived from the equality

$4 b T_{S}+(\alpha+\gamma+b) T_{I}=1$, which is similar to the method for maturation. Solving this

5 for $T_{I}$ gives

6

$7 \quad T_{I}=\frac{\beta_{y} I_{x}}{\left(b+\beta_{y} I_{x}\right)(\alpha+\gamma+b)}$.

9 Now, using (A.4) to find the rates of growth while the invader is in the susceptible

10 and the infected classes gives

11

12

$$
\begin{aligned}
& \rho_{S}=r_{y}-q\left(S_{x}+I_{x}\right), \\
& \rho_{I}=r_{y} k-q k\left(S_{x}+I_{x}\right)-\alpha-(1-k) b .
\end{aligned}
$$

13

14 Combining these in such a way that the fitness function takes the form

15 $s_{x}(y)=\rho_{S} T_{S}+\rho_{I} T_{I}$ gives

16

$$
s_{x}(y)=\frac{1}{b+\beta_{y} I_{x}}\left[r_{y}-q\left(S_{x}+I_{x}\right)+\frac{\beta_{y} I_{x}}{\alpha+\gamma+b}\left(r_{y} k-q k\left(S_{x}+I_{x}\right)-\alpha-(1-k) b\right)\right] .
$$

18

19 In further manipulation based on this quantity (with a trade-off between $r$ and $\beta$ and 20 a non-castrating parasite) we find it convenient to use the quantity 
$1 \quad \theta=S_{x}\left(S_{x}-I_{x}\right)\left(1-\frac{\alpha S_{x}}{\left(S_{x}+I_{x}\right) \sqrt{(r-\alpha)^{2}+4 q \alpha S_{x}}}\right)$

2

3 in the main text.

4 


\section{$1 \quad$ Figure Legends}

3 FIG.1: Example of a singular TIP, displaying the coincidence and mutual tangential 4 property of the trade-off $f$ and two invasion boundaries $f_{1}$ and $f_{2}$ (denoting where

$5 \quad s_{x}(y)=0$ and $s_{y}(x)=0$ respectively) at the tip of the singular TIP (i.e. the top right

6 corner where here $y=x=x^{*}$ ). The evolutionary behaviour in each region, deduced

7 from the evolutionary properties is also shown. The actual evolutionary behaviour

8 exhibited by the evolutionary singularity $x^{*}$ is determined by which region the trade-

9 off curve enters, for example, here the evolutionary singularity is a branching point

10 (the singular point is convergence stable (CS) but not evolutionary stable (ES)). The

11 evolutionary outcome of different trade-off shapes can also be considered. If the

12 curvature of the trade-off was such that it entered the TIP below the $f_{1}$ line then the

13 singular point would be an evolutionary attractor. If the trade-off entered the TIP

14 above the dashed line (the mean curvature of $f_{1}$ and $f_{2}$ ) the singular point would be 15 an evolutionary repellor.

17 FIG. 2: Singular TIPs for a stage-structured single species maturation model. In (A) 18 the trade-off is between the birth rate, $a$, and either the death rate of the juveniles or 19 matures or the intraspecific competition parameter, $b, e$ or $q$ respectively (here $20 f^{\prime}>0$ where $\left.a=f(\cdot)\right)$. In (B) the trade-off is between the birth rate, $a$, and the 21 maturation rate, $m$ (here $f^{\prime}<0$ where $a=f(m)$ ). Note, here, the direction of the 22 TIPs was chosen to give the most biologically direct representation of the trade-off. 
1 FIG. 3: Singular TIPs for a multi-species, single class competition model, with a

2 trade-off between the intrinsic growth rate, $r$, and the competition coefficient

$3 c$ (where $r=f(c)$ and $f^{\prime}>0$ ). Here the cross-species competition rates are linked by

4 the function $c_{z x}=g\left(c_{x z}\right)$ and therefore a change in the competitive ability of one

5 species results in a change in the competitive ability in the other. In (A) this between

6 species interaction is such that an increase in the competitive ability of one species

7 leads to a decrease in the ability of its competitor $\left(g^{\prime}(c)<0\right)$. In (B) the between

8 species competition terms are independent $\left(g^{\prime}(c)=0\right)$. In $(C)$ an increase in the

9 competitive ability of one species leads to an increase in the ability of its competitor

$10 \quad\left(g^{\prime}(c)>0\right)$.

11

12 FIG. 4: Singular TIPs for a multi-class, multi-species host-parasite model (with a non-

13 castrating parasite). Here the trade-off is between the intrinsic growth rate $r$ and the

14 infection rate $\beta$. In (A) the invasion boundaries have curvature (at the tip) such that

15 the mean curvature (dashed line) is positive, and in (B) they have curvature such that

16 the mean curvature is negative.

17

18 


\begin{tabular}{|l|l|l|l|l|}
\hline & $\begin{array}{l}\text { Mature death } \\
\text { rate, } e\end{array}$ & $\begin{array}{l}\text { Maturation } \\
\text { rate, } m\end{array}$ & $\begin{array}{l}\text { Competition } \\
\text { rate, } q\end{array}$ & $\begin{array}{l}\text { Juvenile death } \\
\text { rate, } b\end{array}$ \\
\hline Birth rate, $a$ & $00^{=}$ & $\mathrm{DD}^{=}$ & $00^{=}$ & $00^{=}$ \\
\hline Juvenile death rate, $b$ & $\mathrm{AA}^{=}$ & $00^{=}$ & $00^{=}$ & \\
\hline Competition rate, $q$ & $00^{=}$ & $\mathrm{DD}^{=}$ & & \\
\hline Maturation rate, $m$ & $\mathrm{DD}^{=}$ & & & \\
\hline
\end{tabular}

3 TABLE 1: Shapes of the invasion boundaries on the singular TIP for all the possible

4 choices of parameters for the trade-off for our single species, stage-structured

5 maturation model. The ' $A$ ', ' $D$ ' and ' 0 ' denote whether the invasion boundaries curve

6 in the manner of an accelerating, decelerating or straight trade-off respectively, and $=$

7 denotes the invasion boundaries being identical (superimposed) in which case

8 branching points are not possible. Note that due to the tangential property of the

9 invasion boundaries about the tip of a TIP, linear invasion boundaries will always be

10 superimposed. The left entry represents the invasion boundary $f_{1}$ and the right the

11 invasion boundary $f_{2}$. In all cases strongly accelerating costs lead to attractors while

12 strongly decelerating ones result in repellors, while in DD weak decelerating costs

13 lead to attractors and in AA weak accelerating costs are repellors.

14 


\begin{tabular}{|l|l|l|l|l|}
\hline Interaction & $\begin{array}{l}\text { Sign of the intrinsic } \\
\text { growth of species } x \\
\delta_{1}\end{array}$ & $\begin{array}{l}\text { Sign of the intrinsic } \\
\text { growth of species } z \\
\delta_{2}\end{array}$ & $\begin{array}{l}\text { Sign of the cross species } \\
\text { interaction terms } \\
\delta_{3}\end{array}$ & $\begin{array}{l}\text { Relation between cross- } \\
\text { species interaction terms } \\
c_{z i}, i=x, y\end{array}$ \\
\hline Competition & +1 & +1 & -1 & $g\left(c_{i z}\right)$ \\
\hline Mutualism & +1 & -1 & +1 & $g\left(c_{i z}\right)$ \\
\hline Prey evolution & +1 & +1 & -1 & $-\beta c_{i z}$ \\
\hline Predator evolution & -1 & +1 & $-(1 / \beta) c_{i z}$ \\
\hline
\end{tabular}

2

3 TABLE 2: Sign of the intrinsic growth rate of (evolving) species $x$ (and $y$ ), $\delta_{1}$, and of (fixed) species $z, \delta_{2}$, sign of the cross-species interaction

4 terms, $\delta_{3}$, and the relation, with $c_{i z}$, that the cross-species interaction term $c_{z i}$ takes, for each of our models defined by the dynamics in equation

5 (4), namely competition, mutualism and predator-prey (both prey and predator evolution). 


\begin{tabular}{|l|l|l|l|l|}
\hline & Competition & Mutualism & Prey evolution & Predator evolution \\
\hline $\begin{array}{l}\text { Intrinsic growth rate } \\
\text { against } \\
\text { cross-species interaction } \\
(r \text { vs. } c)\end{array}$ & $0 \mathrm{~A}\left(g^{\prime}>0\right)$ & $0 \mathrm{~A}\left(g^{\prime}>0\right)$ & $0 \mathrm{D}\left(g^{\prime}<0\right)$ & $0 \mathrm{D}\left(g^{\prime}<0\right)$ \\
$\begin{array}{l}\text { OR } \\
\text { Intra-species competition } \\
\text { against } \\
\text { cross-species interaction } \\
(q \text { vs. } c)\end{array}$ & $00^{=}\left(g^{\prime}=0\right)$ & $00^{=}\left(g^{\prime}=0\right)$ & $0 \mathrm{D}\left(g^{\prime}<0\right)$ \\
$\begin{array}{l}\text { Intrinsic growth rate } \\
\text { against } \\
\text { intra-species competition } \\
(r \text { vs. } q)\end{array}$ & $00^{\prime}=$ & $00^{=}$ & $00^{=}$ & $00^{=}$ \\
\hline
\end{tabular}

3 TABLE 3: Shapes of the invasion boundaries on the singular TIP for our multi-species, single class models, for each possible choice of trade-off 4 and possible sign of $g^{\prime}(c)$ for competition and mutualism. The $g^{\prime}(c)$ define how a change in interaction coefficient of one species affects that of 5 the other species. In predator/prey interactions the interaction is always antagonistic $\left(g^{\prime}<0\right)$, but there may be in addition no relationship

$6\left(g^{\prime}=0\right)$ or a positive relationship $\left(g^{\prime}>0\right)$ in both competitive and mutualistic interactions. The 'A', 'D' and ' 0 ' denote whether the invasion 7 boundaries curve in the manner of an accelerating, decelerating or straight trade-off respectively, and = denotes the invasion boundaries being 8 identical (superimposed) in which case branching is not possible. The left entry represents the invasion boundary $f_{l}$ and the right the invasion 9 boundary $f_{2}$. 


\begin{tabular}{|l|l|l|l|}
\hline & $\begin{array}{l}\text { Criterion A: } \\
\text { Trade-off } \\
\text { parameters } \\
\text { appearing in } \\
\text { different } \\
\text { classes/species }\end{array}$ & $\begin{array}{l}\text { Criterion B: } \\
\text { Trade-off } \\
\text { parameters being } \\
\text { characteristics of } \\
\text { different classes }\end{array}$ & $\begin{array}{l}\text { Criterion C: } \\
\text { At least two density } \\
\text { dependent rates } \\
\text { which are } \\
\text { dependent on } \\
\text { different densities }\end{array}$ \\
\hline Type IV TIP & $\checkmark$ & $\checkmark$ & $\checkmark$ \\
\hline Type III TIP & $\checkmark$ & X & $\checkmark$ \\
\hline Type II TIP & $\checkmark$ & $\checkmark$ & X \\
\hline Type I TIP & All remaining combinations & \\
\hline
\end{tabular}

3

4 TABLE 4: Summary of the criteria required to create each of the four types of TIPs.

5 Here type I is two straight invasion boundaries, type II is two curved, superimposed

6 boundaries, type III is one straight and one curved boundary (and therefore

7 separation) and type IV is two curved and separated boundaries.

8 


\begin{tabular}{|l|l|l|}
\hline & $\lambda_{1} \lambda_{2}>0$ & $\lambda_{1} \lambda_{2}<0$ \\
\hline$\lambda_{2} f^{\prime \prime}<\lambda_{2} f_{1}^{\prime \prime}$ & Attractor & Repellor \\
\hline$\lambda_{2} f_{1}^{\prime \prime}<\lambda_{2} f^{\prime \prime}<\lambda_{2}($ mean $)$ & Branching Point & Garden of Eden Point \\
\hline$\lambda_{2} f^{\prime \prime}>\lambda_{2}($ mean $)$ & Repellor & Attractor \\
\hline
\end{tabular}

2

3 TABLE 5: Summary of the evolutionary outcomes for each shape of trade-off (in

4 relation to the invasion boundaries). Here $\lambda_{1}=\operatorname{sig} n\left(\left.\frac{\partial s_{x}(y)}{\partial y_{2}}\right|_{x^{*}}\right)$ (i.e. whether fitness

5 of mutant $y$ increases as we move vertically up a TIP), $\lambda_{2}=\operatorname{sign}\left(\left.\frac{\partial^{2} f_{2}}{\partial y_{1}^{2}}\right|_{x^{*}}-\left.\frac{\partial^{2} f_{1}}{\partial y_{1}^{2}}\right|_{x^{*}}\right)$

6 (i.e. whether the $f_{2}$ boundary is above or below the $f_{1}$ boundary),

$7 \quad$ mean $=\frac{1}{2}\left(\left.\frac{\partial^{2} f_{2}}{\partial y_{1}^{2}}\right|_{x^{*}}-\left.\frac{\partial^{2} f_{1}}{\partial y_{1}^{2}}\right|_{x^{*}}\right)$ (i.e. the mean curvature of the invasion boundaries)

$8 \quad$ and $f_{i}^{\prime \prime}=\left.\frac{\partial^{2} f_{i}}{\partial y_{1}^{2}}\right|_{x^{*}}$ for $i=1,2$

9

10 
1 FIG. 1: HOYLE ET AL.

2

3

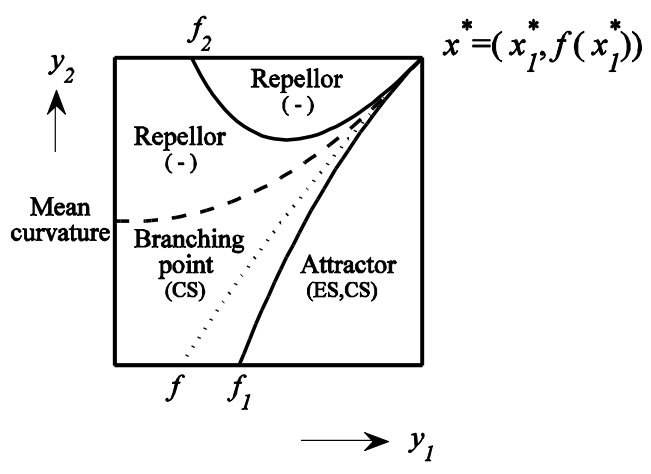


1 FIG 2: HOYLE ET AL.

2

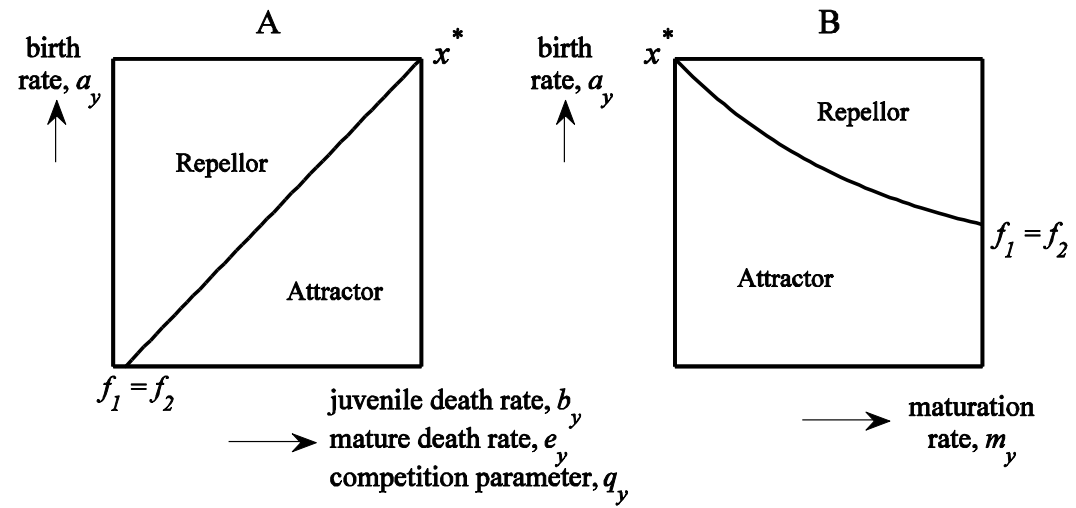


1 FIG 3: HOYLE ET AL.

2

3
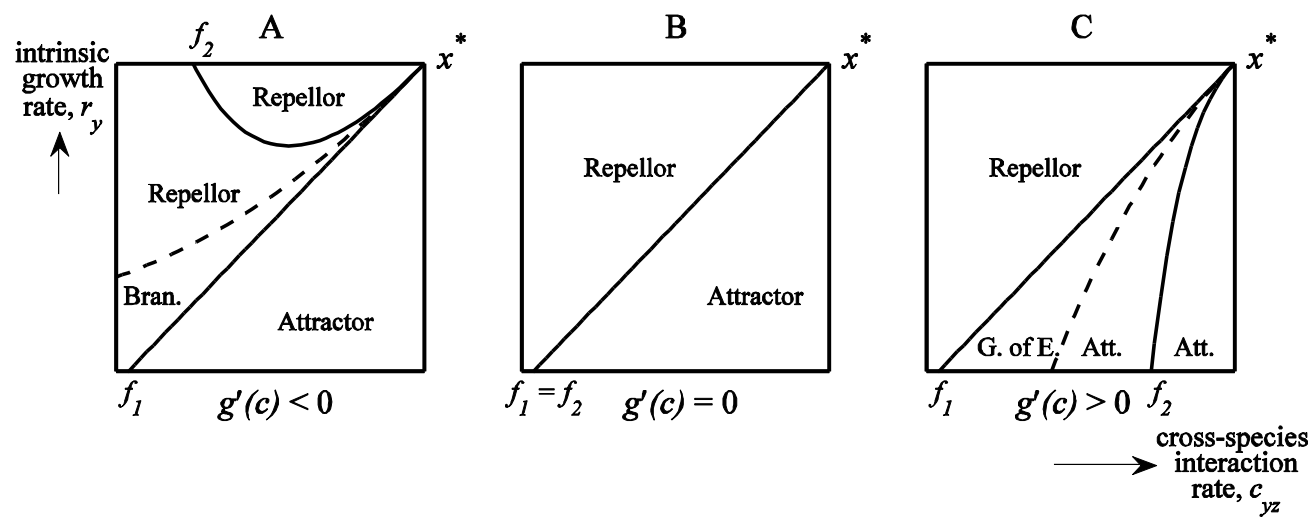
1 FIG. 4: HOYLE ET AL.

2
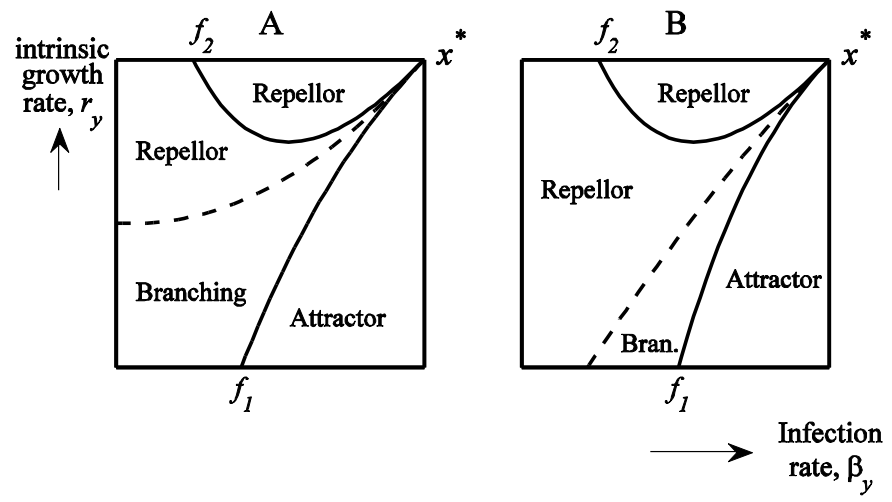